\title{
Analysis of Qualitative Variables in Structural : Models with Unique Solutions
}

\author{
Giorgio Vittadini \\ Università degli Studi di Milano, \\ 20126 - Viale Sarca 202 \\ Milano, Italia, e-mail: vittadin@imiucca.csi.unimi.it
}

\begin{abstract}
A new method based on the Multidimensional Scaling and the Restricted Regression Component Decomposition is proposed in order to obtain solutions for structural models with mixed variables.
\end{abstract}

Keywords: Structural Models with Mixed Variables, Normality Hypothesis, Multidimensional Scaling, Alternating Least Squares, Restricted Regression Component Decomposition.

\section{Structural Model with Qualitative Variables}

The structural model for the study of causal relationship among latent variables is composed of one structural and two measurement equations:

$$
H=H B \div \Xi \Gamma+E=\Xi \Gamma(I-B)^{-1}+E(I-B)^{-1} ; Y=H \Lambda_{y}+U ; X=\Xi \Lambda_{x}+U
$$

where $Y^{\prime}=\left(y^{\prime}(1), \ldots, y^{\prime}(t)\right)\left(n_{y}, t\right), X^{\prime}=\left(x^{\prime}(1), \ldots, x^{\prime}(t)\right)\left(n_{x}, t\right)$ are the observed mixed variables; $\quad \Xi^{\prime}=\left(\xi^{\prime}(1), \ldots, \xi^{\prime}(t)\right)\left(n_{\xi}, t\right), \quad H^{\prime}=\left(\eta^{\prime}(1), \ldots, \eta^{\prime}(t)\right)\left(n_{\eta}, t\right)$ are the latent variables; $E^{\prime}=\left(\varepsilon^{\prime}(1), \ldots, \varepsilon^{\prime}(t)\right)\left(n_{\varepsilon, t}, t\right)$ are the errors in equations; $\Delta^{\prime}=\left(\delta^{\prime}(1), \ldots, \delta^{\prime}(t)\right.$ $\left(n_{\delta} t\right), U^{\prime}=\left(u^{\prime}(1), \ldots, u^{\prime}(t)\right)\left(n_{l} t\right)$, are the errors in variables. It is assumed that: all the random variables have zero mean and finite variance, $B$ is a low matrix with zero on the main diagonal, $(Y, X, H)$ are identically distributed and $(\Xi, E, \Delta, U)$ are identically and independently distributed. The model is usually proposed with restrictions on parameters and on covariances.

The solutions are reached starting from the variance covariance matrix of the reduced model where the variables $H$ in the measurement models are substituted by the value of $\left(\Xi \Gamma(I-B)^{-1}+(I-B)^{-1} E\right)$ obtained from the structural equations. 


\section{Methods for Obtaining Solutions}

First of all given that a continuous bivariate normal variable $\left(J_{l}^{*}, J_{m}^{*}\right)$ with distribution $\Phi\left(j_{i}, j_{m}^{*}, \rho_{\left(j i_{i}, j_{m}\right)}\right)$ underlies every pair of ordinal bivariate observed variables $\left(J_{1}, J_{m}\right)$, the polychoric correlation between the two components of them is calculated. When there is one only ordinal variable, the polyserial correlation coefficient between an observed quantitative variable and the normal underlying variable is defined. The observed frequencies of the qualitative variables $n_{j_{q_{q}} j_{m_{r}}}\left(q=1, \ldots n_{i} ; r=1, \ldots, n_{r}\right)$ given, the polychoric coefficients are reached in different ways. Jöreskog (1994) hypothesizes that the marginal probability of the normal variables are equal to the marginal frequencies of the two-way table $\left(\pi_{j_{q},}=n_{j_{q} .}\right)\left(\pi_{j_{r_{*}}}=n_{j_{m} .}\right)$ of the ordinal variables. Therefore, first of all, he reaches the thresholds, using the marginal distributions of the normal bivariate distribution; for given thresholds using such distribution, he obtains the correlation coefficient maximizing the log likelihood of the sample respect to $\rho_{\left(j ;, j_{m}\right)}$. Lee et al. (1990) estimate ine thresholds by means of Partition Maximum Likelihood (P:ML) which is simpler from the computational point of view.

Lee et al. (1995) estimate simultaneously the correlation coefficients and the thresholds concerning pairs of variables maximizing the log likelihood of the sample respect to $q_{\left(i_{i}, j_{m}\right)}$ and respect to every threshold $j_{q_{q}}, j_{m}$, by means of PML (but Jöreskog (1994) observes that "different estimates of thresholds for one variable may be obtained from different pairs of variables").

By means of Full Maximum Likelihood, in one case Lee et al. (1992) simultaneously reach all the thresholds of the polychoric correlations; in another case (Lee et al. (1990)), they reach also the parameters, the variances and the covariances of the latent variables. Moreover these two last methods take up too much computer time (Lee et al. (1990) Lee et al. (1995)).

In the first three methods, the parameters and the covariances of the latent variables and errors are obtained from the polychoric or polyserial correlations. There is not a unique understanding about the method of obtaining the parameters and the latent variables. Jöreskog (1990), (1994), Rigdon and Ferguson (1991) propose the Weighted Least Squares method and criticise the Maximum Likelihood method because the standard error parameter estimates are asymptotically incorrect, but Lee et al. (1995) continue to prefer the General Least Squares method and criticize the proposal of Jöreskog because it requires sample sizes larger than 200 and more computer time. 


\section{Some Critical Observations}

Comparing, in some Montecarlo studies, different correlatin coefficients when the underlying bivariate distribution is normal, the poly oric correlation is shown to be "the best in the sense of being closest to the al. 987) show (Quiroga (1992)) [even if in a Montecarlo study Babak of model ; arameters and the polychoric coefficient provides the best (1984), Aish and Jöreskog (1990) and the worst fit statistics]. However, Muthen (10 assumption of underlying bivariate normality Quiroga (1992) say also that "the assumplon of un social sciences". There are not is too strong for most ordinary variables usefficient with underlying not normal many studies on the use of polychoric coefrobustness of polychoric coefficient distribution. Lee and Lam (1988) sistribution is elliptical (containing multivariate only when the underlying distribution is etributions). Lee et al. (1995) even nomal, platicentric and leptocentric distibutions) huch robustness with moderate though obtaining quite satisfactory results aboun definite conclusion, a longer size random samples, say that "to draw literaiure only Quiroga (1992) tries, in a simulation is needed". Moreover in literature ony Qupothesis of the continuous systematic way, to extend the distribubles. First of all, she says that when variables underlying the qualitative varables Fariables are surely noi consistent jou leave the normality assumptions, Noreover, in a Monteca:lo study she and slightly biased (Quiroga (192)). Mistribution is a skew-normal bivariate, the verifies that, when the underlying distice only for sample size of 200-400 and polychoric coefficient is the best cho and underestimates the true correlation large number of categories coefficient. Then, by means of a meare generated by the Fleishman-Valewhen the underlying distributions are gemation departure from normality due to Maurelli polynomial transformation (wic coefficient is robust, but overestimates skewness and kurtosis) the polychoric coes an extended polychoric coefficient the true correlation. Finally she propor a normal and univariate skew-normal with distribution given by a mixture om a nirical verifications. Therefore, until density function but she does not givemonstrations nor empirical simulations now, there are neither theoretical dencrly valid reasons for using polychoric which give satisfactory and gener all distribution. coefficient with underlying not nom polychoric coefficients: are not sensible to Moreover the solutions based varables because they generally deal only with different scale of qualitative variablem variance covariance-covariance matrix ordinal variables, reach solutions from the solutions obtained from the observed of the reduced model different from models and have the same problems of variables of the original measurement indeterminacy of latent variables of

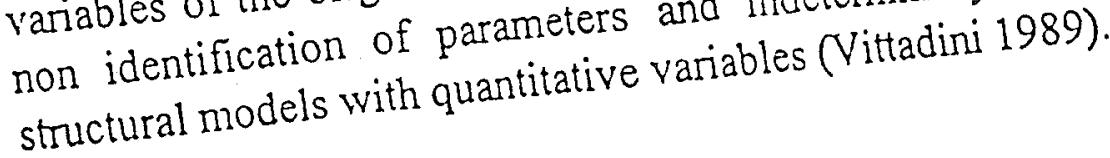




\section{An Alternative Proposal}

In the quantitative case the problems of not uniqueness of the solutions are resolved by using linear combinations instead of causal latent variables of the observed variables (Wold (1982), Haagen and Vittadini (1991)). In this paper we propose to obtain the latent variables of the model as linear combinations of the observed mixed variables simultaneously quantified, by means of methods of multidimensional scaling using simultaneously optimal scaling and ordinary least squares method. In fact such methods resolve the problem of not normality of the variables because are distribution free (Young (1981)) and give unique solutions once chosen the method of multidimensional scaling. In order to avoid subjective choices about methodologies of multidimensional scaling (and therefore subjective solutions), we propose: to quantify the qualitative variable and to obtain the linear combinations of them by means of a unique objective function; to reach flexible solutions as regards to different kinds of linear combinations requested by the problems; to take into account the different scale of the qualitative variable. Therefore, among the variety of multidimensional scale methods we choose the family of Alsos method (Young (1951), and Keller and Wansbeek (1983)). These methods are based on altemating optimal scaling which quantifies qualitative variables and ordinary least squares which reach linear combinations of them in a iterative way. So they obtain solutions in a different way along the scale of the variables (ordinal-nominal, continuousdiscrete), and the aim of analysis (e.g. Principal components, canonical correlation) giving answers to previous problems. Among the family of Alsos methods we avoid methods such as OSMOD (Saito and Otsu (19SS)) or INDOMIX-CAMIX (Kiers (1991)) which obtain solutions in tịo stages. Instead we choose methods that simultaneously obtain quantifications of qualitative variables and their linear combinations (ADDALS (De Leeuw, Young, Takane (1976)) MORALS CORALS (Young, De Leeuw Takane, (1976)), PRNCALS ( De Leeuw and Van Rijckevorsel (1980)) OTERALS (Van Der Burg and De Leeuw (1988)) respectively from the perspective of variance analysis, canonical correlation, principal components, multiple correspondence analysis. Moreover in order to obtain the latent variables from their real indicators as in Wold (1982), we apply the chosen Alsos methods to the subsets of mixed variables $Y_{\beta}, X_{\delta}$ characterized by submatrices $l_{y(\beta .0)}, l_{x(\delta, 0)}$ with coefficients all different from zero. So we simultaneously obtain the quantification $Y_{\beta}^{*}, X_{\delta}^{*}$ of such mixed variables $Y_{\beta}, X_{\delta}$ and their linear transformations $\tilde{\eta}_{\beta}, \tilde{\xi}_{\delta}$ according to different aim of the analysis (e.g. canonical correlation, principal analysis etc.). Then in order to take into account the restrictions: 


$$
\begin{aligned}
& \operatorname{cov}\left(\eta_{\beta}, \eta_{x}\right)=0 ; \operatorname{cov}\left(\xi_{\delta}, \xi_{\gamma}\right)=0 ; \operatorname{cov}\left(\delta_{\beta_{2}}, \delta_{\beta_{3}}\right) ; \operatorname{cov}\left(u_{\delta_{2}}, u_{\delta_{\mu}}\right)=0 \\
& b_{(\beta, \mu)}=0 ; \gamma_{(\delta, \varphi)}=0 ; l_{y(\beta, \alpha)}=0 ; l_{x(\delta, v)}=0
\end{aligned}
$$

we obtain by means of the Restricted Regression Component Decomposition (RRCD) of quantified variables $Y^{*}, X^{*}$ (Haagen and Vittadini (1998)) by means of an iterative process:

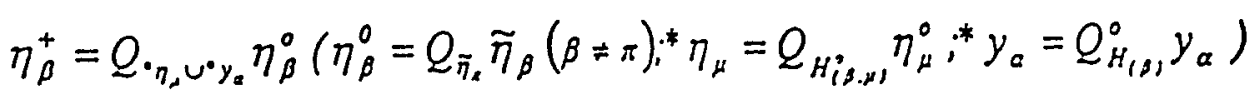

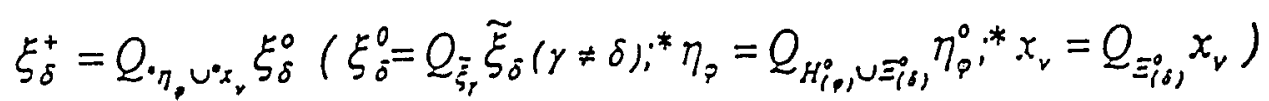

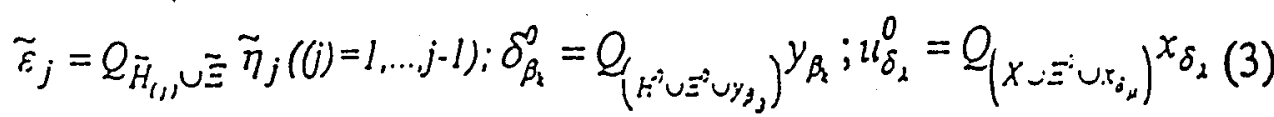

where $H_{(\mu, \beta)}^{\circ}$ are the $H^{*}$ without $\eta_{\mu}^{\circ}, \eta_{\beta}^{\circ}, Q_{\pi_{i}}$ is the complement orthogonal to the orthogonal projector on the space generated by $\tilde{\eta}_{\pi}$ and the other symbols are defined in a similar way. So we have the following RRCD of $y_{\beta_{1}}, x_{\delta_{\alpha}}, \eta_{\beta}^{0}$ :

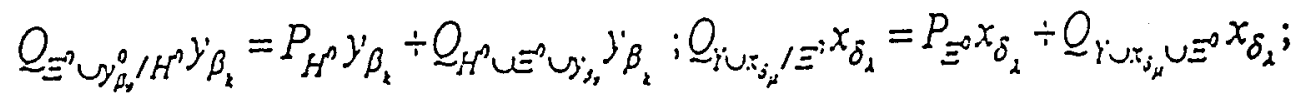

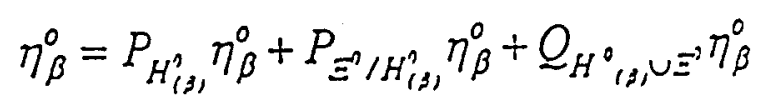

\section{Numerical Example}

The following variables are observed on a sample of 150 families casually chosen from 4103 american families that have been codified in the Federal Reserve Board research regarding National Income and Wealth of 1983.

$Y_{1}$ Job contract household $\left(y_{11}\right)$, spouse $\left(y_{12}\right)$; Occupation kind household $\left(y_{13}\right)$, spouse $\left(y_{14}\right)$; Occupation sector household $\left(y_{15}\right)$, spouse $\left(y_{16}\right)$. $Y_{2}$ Total health $\left(y_{21}\right)$; Income $\left(y_{22}\right)$; Debt $\left(y_{23}\right)$. $X_{1}$ Age household $\left(x_{11}\right)$, spouse $\left(x_{12}\right)$; Sex household $\left(x_{13}\right)$, spouse $\left(x_{14}\right)$; Number of children $\left(x_{15}\right)$; Race $\left(x_{16}\right)$; Residence region $\left(x_{17}\right)$; Civil Status $\left(x_{18}\right) ; X_{2}$ Educational Level household $\left(x_{21}\right)$, spouse $\left(x_{22}\right)$; Full time job years household $\left(x_{23}\right)$, spouse $\left(x_{24}\right)$; Part time job years household $\left(x_{25}\right)$, spouse $\left(x_{26}\right)$; Latent variables: labour force $\left(\eta_{1}\right)$; Health and income $\left(\eta_{2}\right)$, Civil status $\left(\xi_{1}\right)$, Instruction grade $\left(\xi_{2}\right)$.

In order to verify the causal dependence of the latent variables $\mathrm{H}$ from the latent variables $\Xi$ we use the alternative proposal shown in paragraph 4 with the

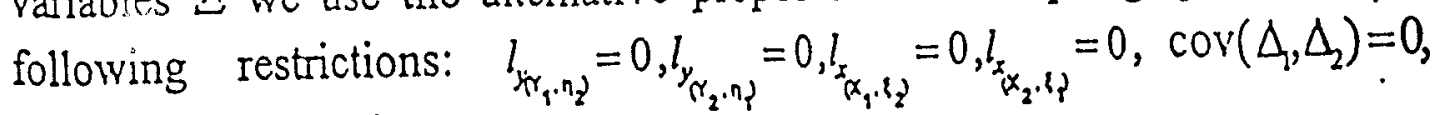
$\operatorname{cov}\left(U_{1}, U_{2}\right)=0$. The qualitative variables are quantified and the latent variables 
are obtained as principal components with Princals method, the restrictions are then taken into account by means of RRCD. The variance-covariance of the observed variables and the results are shown in table 1.

Table 1: The alternative proposal for the sample of American families.

\begin{tabular}{|c|c|c|c|c|c|c|c|c|}
\hline & 0.907 & -0.0235 & 0.02 .18 & $\begin{array}{c}S_{f} \\
-0.0017\end{array}$ & 0.0580 & 19.85s & -0.0163 & -0.078 \\
\hline .0281 & 8.2171 & -1.2383 & 0.0211 & 0.8073 & $-0.358 s$ & -77.107 & 12.671 & 0.5565 \\
\hline coss & -3.2353 & 17.0838 & $-2.03 / 3$ & -0.6388 & 4.1928 & -3.3 .60 & $-17.4: 3$ & -1.1561 \\
\hline 0.0288 & 0.0917 & -0.2313 & $0.1+30$ & 0.0187 & -0.0858 & 80.287 & 8.5693 & -0.0821 \\
\hline-0.0049 & 03073 & -0.5313 & 0.0107 & 2.5353 & -0.7303 & $-15: .13$ & 15.437 & 0.1698 \\
\hline 0.0580 & -0.3535 & 1.1238 & -20853 & -0.7303 & 11.735 & -65.833 & -87.510 & 0.1070 \\
\hline 10.353 & $-73.10 \%$ & $-323.6 \div 5$ & 89.247 & $-0.215 !$ & -65.533 & Jas?:i & 1:08: & -337.51 \\
\hline- & 12.001 & $-17.8: 5$ & 85830 & 1548 & -37.510 & dEs?:1 & $1311 t$ & -21.173 \\
\hline & (sss] & $1.18 \leqslant 1$ & -2.0821 & $0+568$ & 0.1070 & - 3riss & - illil? & 7.4133 \\
\hline
\end{tabular}

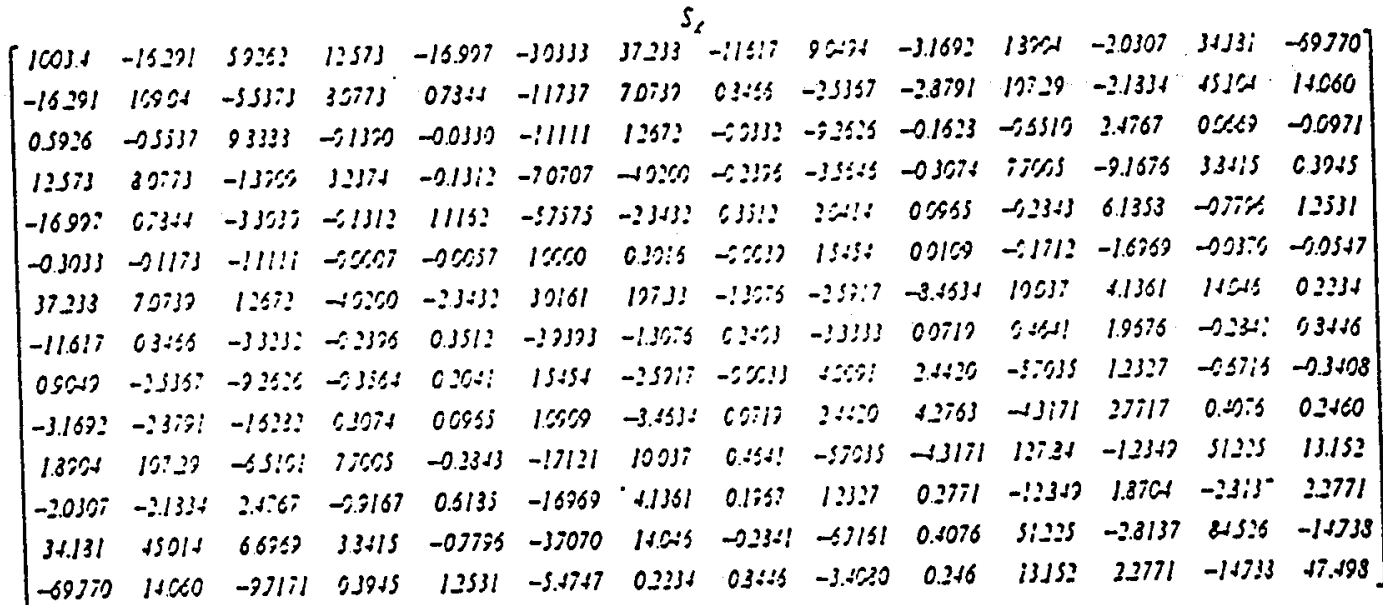

$\left[\begin{array}{cc}0 & -0.0962 \\ -0.6757 & 0\end{array}\right]$
$\left[\begin{array}{cc}0.0171 & 0 \\ 0 & 0.0022\end{array}\right]$

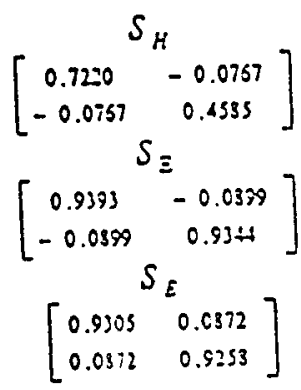

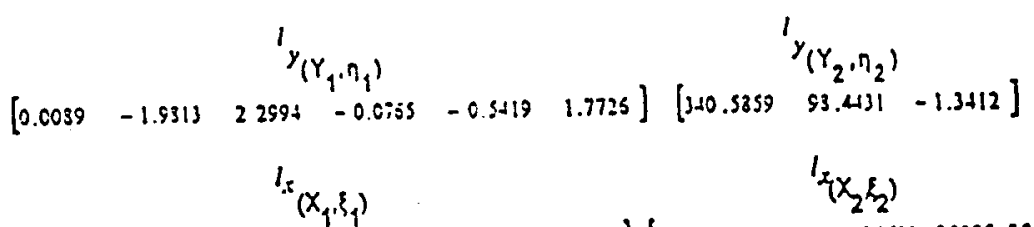

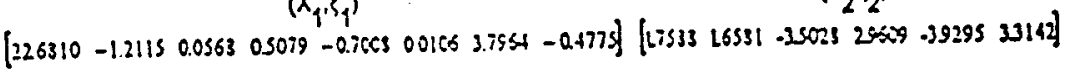

$$
S_{d_{1}}
$$

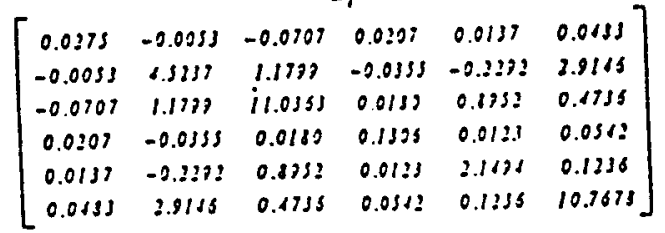

$S_{U_{1}}$

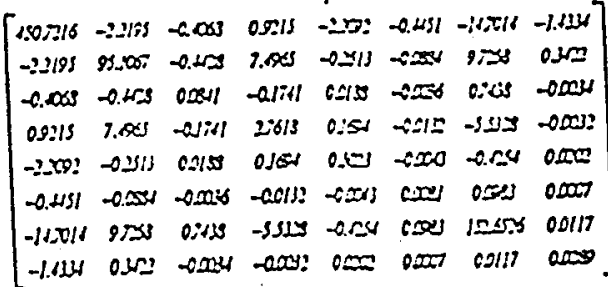

$S_{S_{1}}$

$\left[\begin{array}{ccc}113117.7 & 61569 & 118.6 \\ 61567 & 7017.8 & 81.98 \\ 118.6 & 81.83 & 3.412\end{array}\right]$

$S_{U_{3}}$

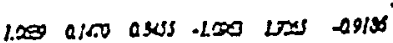

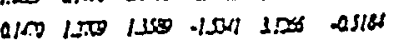

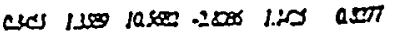
L100

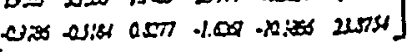

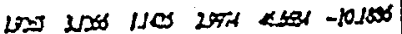

With the example we can verify that the altemative proposal respects all the properties of the structural model described in paragraph 1 and the restrictions indicated in this paragraph. But the alternative proposal obtains unique 
solutions solving all the problems of non-identification of parameters and indeterminacy of latent variables of the structural models with qualitative variables.

\section{References}

Aish, A. M., Jöreskog, K. G. (1990). A panel model for political efficacy and responsiveness: an application of LISREL7 with weighted least squares, Quality and Quantity, 24, 405-426.

Babakus, E., Ferguson, C.E. Jr, Jöreskog K.G. (1987). The sensitivity of confirmatory maximum likelihood factor analysis to violations of measurement scale and distributional assumptions, Journal of Marketing Research, 24, 222-228.

De Leeuw, J., Van Rijckevorsel, J.(1980). Homals \& princals, some generalizations of principal components analysis, In E. Diday et al. (Eds.), Data Analysis and Informatics, North-Holland Publishing Company, 231241.

De Leeuw, J., Young, F.W., Takane Y. (1976). Additive structure in qualitative data: an alternating least squares method with optimal scaling features, Psychometrika, 41, 471-503.

Haagen, K., Vittadini, G. (1991). Regression Component Decomposition in Structural Analysis, Communications in Statistics, 20, 1153-1161.

Haagen, K., Vittadini, G. (1998). Regression Component Decomposition Restricted. Un'alternativa al Lisrel model, Metron, 56, 1-2, in corso di pubblicazione.

Jöreskog, K.G. (1990). New developments in Lisrel: analysis of ordinal variables using polychoric correlations and weighted least squares, Quality and Quantity, 24, 387-404.

Jöreskog, K.G. (1994). On the estimation of polychoric correlations and their asymptotic covariance matrix, Psychometrika, 59, 3, 381-389.

Kiers, H. A. L. (1991). Simple structure in component analysis techniques for mixtures of qualitative and quantitative variables, Psychometrika 56, 2, 197212.

Keller, W. J., Wansbeek, T. (1983). Multivariate methods for quantitative and qualitative data, Journal of Econometrics, 22, 91-111.

Lee, S.Y, Lam M. L.(1988). Estimation of polychoric correlation with elliptical latent variables. Journal of statistic Computation and Simulation, 30, 173188.

Lee, S.Y., Poon, W.Y., Bentler, P.M. (1990). Full maximum likelihood analysis of structural equation models with polytomous variables, Statistics and Probubility Letters, 9, 91-97. 
Lee, S.Y., Poon, W.Y., Bentler, P.M. (1995). A two-stage estimation of structural equation models with continuous and polytomous variables, British Joumal of Mathematical and Statistical Psychology, 48, 339-358.

Muthen, B. (1984). A general structural equation model with dichotomous, ordered categorical, and continuous latent variable indicators, Psychometrika, 49, 1, 115-132.

Quiroga, A.M. (1992). Studies of the Polychoric Correlation and other Correlation Measures for Ordinal Variables, $\mathrm{PhD}$ thesis, Uppsala University.

Rigdon, E.E., Ferguson, C.E. Jr. (1991) The performance of the polychoric correlation coefficient and selected fitting functions in confirmatory factor analysis with ordinal data, Journal of Marketing Research, 28, 491-497.

Saito, T., Otsu, T. (1988). A method of optimal scaling for multivariate ordinal data and its extensions, Psychometrika, 53, 1, 5-25.

Van Der Burg, E., De Leeuw, J.(1988). Homogeneity analysis with $k$ sets of variables: an altemating least squares method with optimal scaling features, Psychometrika, 53,2, 177-197.

Vittadini, G. (1989). Indeterminacy Problems in the LISREL Model, in Multivariate Behavioral Research, Forth Worth (Texas), 24, 4, 397-414.

Wold, H. (1982). Soft Modelling: the basic design and some extensions, in Jöreskog K.G., Wold H., Systems under indirect observation: casualit); structure, prediction, North - Holland, Amsterdam, 2, 1-54.

Young, F.W. (1981). Quantitative analysis of qualitative data, Psychometrika, 46, 357-388.

Young, F.W., De Leeuw, J., Takane, Y. (1976). Regression with qualitative and quantitative variables: an alternating least squares method with optimal scaling features, Psychometrika, 41, 4, 505-529. 